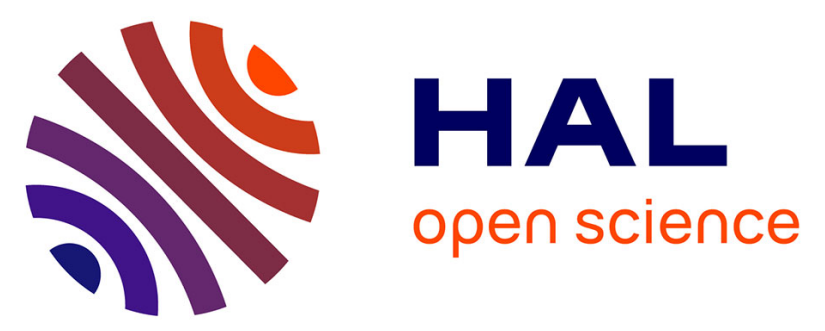

\title{
FISH-quant: automatic counting of transcripts in 3D FISH images
}

\author{
Florian Mueller, Adrien Senecal, Katjana Tantale, Hervé Marie-Nelly, \\ Nathalie Ly, Olivier Collin, Eugenia Basyuk, Edouard Bertrand, Xavier \\ Darzacq, Christophe Zimmer
}

\section{To cite this version:}

Florian Mueller, Adrien Senecal, Katjana Tantale, Hervé Marie-Nelly, Nathalie Ly, et al.. FISH-quant: automatic counting of transcripts in 3D FISH images. Nature Methods, 2013, 10 (4), pp.277 - 278. 10.1038/nmeth.2406 . pasteur-01622707

\section{HAL Id: pasteur-01622707}

https://hal-pasteur.archives-ouvertes.fr/pasteur-01622707

Submitted on 24 Oct 2017

HAL is a multi-disciplinary open access archive for the deposit and dissemination of scientific research documents, whether they are published or not. The documents may come from teaching and research institutions in France or abroad, or from public or private research centers.
L'archive ouverte pluridisciplinaire HAL, est destinée au dépôt et à la diffusion de documents scientifiques de niveau recherche, publiés ou non, émanant des établissements d'enseignement et de recherche français ou étrangers, des laboratoires publics ou privés.

\section{(2)(1) $\$$}

Distributed under a Creative Commons Attribution - NonCommercial - ShareAlikel 4.0 


\section{FISH-QUANT: automatically counting transcripts in 3D FISH images}

Florian Mueller ${ }^{1,2}$, Adrien Senecal ${ }^{2}$, Katjana Tantale ${ }^{3}$, Hervé Marie-Nelly ${ }^{1}$, Nathalie Ly ${ }^{2}$, Olivier Collin ${ }^{2}$,

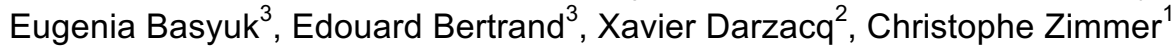

${ }^{1}$ Institut Pasteur, Unité Imagerie et Modélisation, Centre National de la Recherche Scientifique, Unité de Recherche Associée 2582, 25-28 rue du Docteur Roux, 75015 Paris, France

2 Institut de Biologie de l'Ecole Normale Supérieure, Functional Imaging of Transcription, Centre National de la Recherche Scientifique, Unité Mixte de Recherche 8197, 46 rue d’Ulm, 75005 Paris, France

${ }^{3}$ Institut de Génétique Moléculaire de Montpellier, Centre National de la Recherche Scientifique, Unité Mixte de Recherche 5535, 34293 Montpellier Cedex 5, France

Correspondence to: Edouard Bertrand (edouard.bertrand@igmm.cnrs.fr), Xavier Darzacq (darzacq@ens.fr), Christophe Zimmer (czimmer@pasteur.fr)

General comment:

The figure is too large, it alone will take half a print page if not more making the text a tight fit on two pages. We cannot go over two pages.

Transcription is inherently stochastic even in clonal cell populations ${ }^{1}$. Studies at single-cell-singlemolecule level enable a quantitative understanding of the underlying regulatory mechanisms $\mathrm{s}^{2,3}$. A widely used technique is single-molecule RNA fluorescence in-situ hybridization (FISH), in which fluorescent probes target the mRNA of interest and individual molecules appear as bright diffraction-limited spots $(\text { Fig. 1a,b })^{3}$. Recent experimental progress makes FISH easy to use ${ }^{4}$, but a dedicated image analysis tool is currently missing. Available methods allow counting of isolated mature mRNAs but cannot reliably quantify the dense mRNA aggregates at transcription sites (TS) in three dimensions (3D), particularly of highly transcribing genes ${ }^{4}$. We developed FISH-QUANT to close this gap (Supplementary Note 1).

FISH-QUANT first detects, then localizes mature mRNA in 3D by fitting Gaussians to fluorescent spots ${ }^{5}$; each mRNA then undergoes a quality test based on the measured point spread function (PSF) (Fig. 1b and Supplementary Note 2). This provides the 3D position of mRNAs inside cells and the distribution of mRNA counts across the cell population (Fig. 1b). We validated this method in simulations and experimentally by dual-color FISH against RBP1 where we obtained excellent agreement in number of estimated spots and co-localization (Fig. 1c-e, Supplementary Note 3).

Counting nascent mRNA is more challenging because their high local density prevents resolving individual molecules. FISH-QUANT offers two solutions: (i) comparison of the integrated intensity of the TS to that of mature mRNA, (ii) reconstruction of the TS signal by iterative superposition of weighted PSFs $^{5}$ (Supplementary Note 4). Whereas simple methods such as comparing maximum peak intensities ${ }^{6}$ ignore the 3D extent of the TS, FISH-QUANT explicitly accounts for it. Also, method (ii) uses the experimentally measured PSF and restricts the intensity of the weighted PSFs to the range observed for mature transcripts, thereby considering aberration effects and variable labeling efficiency. Note that FISH-QUANT estimates an equivalent number of fully elongated transcripts; different positioning of the FISH probes on the mRNA affects the results and can be used to infer important properties of transcription (Supplementary Note 4). To validate these methods, we first used simulations (Supplementary Note 5). For diffraction-limited TS, all methods yielded accurate estimates (Fig. 1f). 
However, for larger TS, the simple methods led to gross underestimates, whereas FISH-QUANT still gave accurate results (Fig. 1f). For elongated TS only the PSF superposition approach worked reliably. For experimental validation, we used an artificial reporter with TS frequently exceeding the diffraction limit. An RNAse protection assay (RPA) provided a rough, but independent estimate of the ratio of mature vs. nascent mRNA. RPA yielded ratios within the same range as the FISH-based quantifications, confirming their general validity (Supplementary Note 6). For a more accurate assessment of simple methods and FISH-QUANT, we then compared the nascent transcript counts. As for large simulated TS, the simple methods led to underestimates (Fig. 1g). Thus, FISH-QUANT accurately quantifies nascent mRNA when simple approaches do not. Finally, we analyzed $\beta$-actin mRNA after serum induction and measured more than twice the amount of nascent mRNA than simple methods, illustrating the importance of accurate quantification even for endogenous genes (Supplementary Note 6). Finally, note that FISH-QUANT could also be applied to other structures with a dense accumulation of mRNA such as P-bodies or stress granules.

FISH-QUANT is controlled via graphical user-interfaces in Matlab and requires no computational expertise. A batch mode allows to automatically process multiple images. FISH-QUANT is available at http://code.google.com/p/fish-quant/ with a detailed manual and test data.

\section{Acknowledgments}

This research was supported by Agence Nationale de la Recherche (ANR 2010 BLAN 1222 01, ANR-09PIRI-0024, 2010-INTB-1401-02), Sidaction, Région Ile-de-France in the framework of C'Nano IdF (The nanoscience competence center of Paris Region), Fondation pour la Recherche Médicale en France (FRM) and Institut Pasteur. We thank Jim McNally for editing of the manuscript.

\section{References}

1. Li, G.-W. \& Xie, X. S. Nature 475, 308-315 (2011).

2. Zenklusen, D., Larson, D. R. \& Singer, R. H.. Nat. Struct. Mol. Biol 15, 1263-1271 (2008).

3. Itzkovitz, S. \& Van Oudenaarden, A. Nat Meth 8, S12-S19 (2011).

4. Raj, A., Van den Bogaard, P., Rifkin, S. A., Van Oudenaarden, A. \& Tyagi, S. Nat Meth 5, 877-879 (2008).

5. Thomann, D., Rines, D. R., Sorger, P. K. \& Danuser, G. J Microsc 208, 49-64 (2002).

6. Boireau, S. et al. J. Cell Biol. 179, 291-304 (2007). 

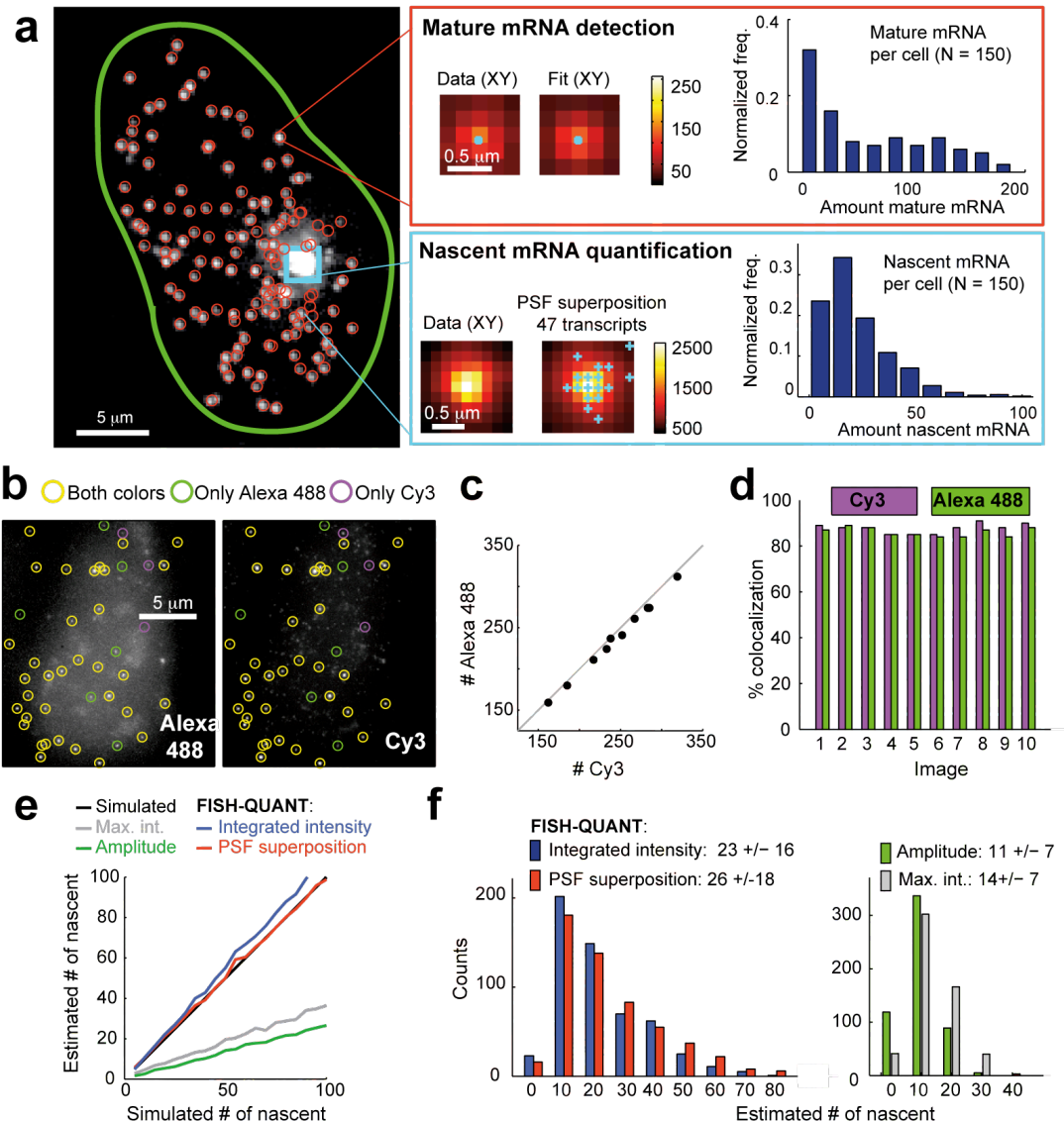

Figure 1. Counting mature and nascent mRNA in FISH-QUANT. (a) FISH image of Hygro-MS2-x96-bGH reporter (Supplementary Note 6). Cell (green outline) with detected mRNA (red circles) and transcription site (blue box). Red box: individual mRNA (left) is fit with 3D Gaussian (right), yielding localization (blue dot) with sub-pixel accuracy. Blue box: transcription site is quantified using PSF superposition. Histograms show distribution of nascent and mature mRNA per cell, obtained by batch processing of 150 cells. (b) Validation of mature mRNA detection by dual color FISH against RBP1. Left: detection with 15 Alexa 488 probes, right: with 15 Cy3 probes. (c) Spot counts from two colors in 11 analyzed images. (d) Co-localization in each image. (e) Validation of transcription site in simulations. Left: for diffraction-limited sites, all methods yield accurate quantification. Right: For spatially extended sites, simple methods underestimate mRNA counts, whereas FISH-QUANT provides accurate estimates. (f) Transcription site quantification of Hydro-MS2x96-bGH with FISH-QUANT methods (left) and simple methods (right). 